AIP Appilied Physics

\title{
Phaseresolved photoacoustic microscopy: Application to ferromagnetic layered samples
}

C. L. Cesar, H. Vargas, J. Pelzl, and L. C. M. Miranda

Citation: J. Appl. Phys. 55, 3460 (1984); doi: 10.1063/1.332954

View online: http://dx.doi.org/10.1063/1.332954

View Table of Contents: http://jap.aip.org/resource/1/JAPIAU/v55/i10

Published by the American Institute of Physics.

\section{Additional information on J. Appl. Phys.}

Journal Homepage: http://jap.aip.org/

Journal Information: http://jap.aip.org/about/about_the_journal

Top downloads: http://jap.aip.org/features/most_downloaded

Information for Authors: http://jap.aip.org/authors

\section{ADVERTISEMENT}

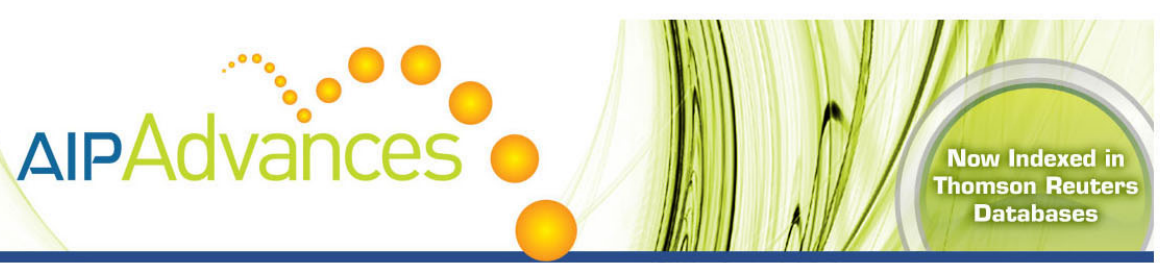

\section{Explore AIP's open access journal: •Rapid publication \\ - Article-level metrics \\ - Post-publication rating and commenting}




\title{
Phase-resolved photoacoustic microscopy: Application to ferromagnetic layered samples
}

\author{
C. L. Cesar, H. Vargas, and J. Pelz|a) \\ Instituto de Física, Universidade Estadual de Campinas, 13100, Campinas, SP, Brazil \\ L. C. M. Miranda \\ Instituto de Estudos Avançados, Centro Técnico Aeroespacial, 12200, S. J. Campos, SP, Brazil
}

(Received 29 September 1983; accepted for publication 1 February 1984)

\begin{abstract}
The possibility of using the phase shift of the acoustic signal of different constituents as a new tool for a depth profile analysis of layered samples is discussed. This new method is experimentally demonstrated by measuring the phase variations of the photoacoustic detected ferromagnetic resonance of a sample consisting of an iron film bonded to a nickel film.
\end{abstract}

PACS numbers: 43.35.Sx, 76.50. $+\mathrm{g}, 78.20 . \mathrm{Hp}, 68.60 .+\mathrm{q}$

Since the first demonstration of the photoacoustic (PA) effect in the microwave region of the spectrum by Diebold and $\mathrm{McFadden}{ }^{1}$ using gaseous $\mathrm{O}_{2}$, a number of authors ${ }^{2-10}$ have devoted themselves to the investigation of the PA effect in solid samples using microwaves. In this way, the PA detection of ferromagnetic resonance of metallic samples ${ }^{2}$ as well as the paramagnetic resonance of several organic and (or) inorganic substances ${ }^{3-10}$ have been reported. In particular, it has been demonstrated by Netzelmann et al., ${ }^{5}$ when studying the PA detected paramagnetic resonance of highspin ferri-tetraphenylporphyrin [Fe(III)-TPP], that it is possible to distinguish between different paramagnetic species by measuring the phase shift of their acoustic response to the microwave absorption. The usefulness of the phase-lag measurements in PA spectroscopy has been recognized since 1977 by Adams and Kirkbright ${ }^{11}$ and more recently by Cesar $e t a l,{ }^{12}$ as a tool for monitoring the thermal diffusivity.

In this paper we explore the possibilities of using the phase shift of the acoustic signal of different constituents as a tool for a depth profile analysis of layered samples (i.e., for PA microscopy). The basic idea may be summarized as follows. Let us consider a typical PA arrangement in which heat is generated within the sample due to the absorption of chopped radiation. For the sake of the argument, we further assume that the sample is made of two layers of materials $A$ and $B$ with material $A$ facing the gas phase of the PA cell. At a fixed modulation frequency, the acoustic signal detected at the microphone is the resultant of the contributions of the heat generated in both constituents $A$ and $B$. As the component $B$ is beneath $A$ there should exist a time lag between the signals arising from $A$ and $B$ due to the difference in the corresponding thermal diffusion times. This difference in the time lengths to reach the gas phase produces a phase shift $\theta$ between the two signals. Thus, the actually observed signal $S$ may be viewed as the resultant of two vectors (whose lengths $S_{A}$ and $S_{B}$ correspond to the signals of $A$ and $B$, respectively) with an angle $\theta$ between them. This means that once the angle $\theta$ is known, by varying the phase of $90^{\circ}$, say with respect to the signal $S_{A}$, one should observe only the contribution of component $B$, and vice versa. In other words, by

\footnotetext{
a) Permanent address: Ruhr, Universität Bochum, Abteilung für Physik und Astronomie, D-4630 Bochum, FRG.
}

measuring the phase variation of the PA signal of a composite sample one may, in principle, single out the contribution of the different constituents at different locations. This alternative detection technique differs from that used in conventional PA microscopy. ${ }^{13}$ Commonly, the depth profiling analysis is carried out by measuring the PA signal at different modulation frequencies. Subsurface locations are then differentiated by the change of the penetration depth of the heat wave (i.e., the thermal diffusion length) with the modulation frequency. The alternative technique suggested above, in contrast, measures the PA signal at a constant modulation frequency at various phase angles; i.e., the different subsurface layers are identified by the transit time of the heat generated at these locations. Phase-resolved plots can be obtained by two procedures. In one case, the in-phase component of the PA signal is recorded several times, changing the phase angle offset. This procedure has been used by Mackenthun $e t$ $a l .{ }^{14}$ when investigating pigment distribution in lobster shells. The alternative technique we propose here does not require several experimental runs as in Ref. 14. By measuring the in-phase and the out-of-phase components simultaneously, we show, in what follows, that the phase-resolved plots are readily obtained by means of calculation.

To demonstrate the usefulness of this latter technique for the PA microscopy we have conducted PA detection of ferromagnetic resonance of a layered sample made of a nickel film bonded to an iron film both of $50-\mu \mathrm{m}$ thickness. The experimental arrangement is shown schematically in Fig. 1, where the sample is placed at the shorted end of a waveguide driven by an $X$-band $(8-12.4 \mathrm{GHz})$ source delivering $10 \mathrm{~mW}$ of power. The microwave power was modulated with a lockin signal. The sample was then followed by the detection gas cell in which a Bruel and Kjaer (BK-4166) condenser microphone is mounted on one of its wall. A second cell was also mounted in one of the cavity's wall. This second cell was built by drilling the cavity's wall. In the inner face we covered the hole with an aluminum foil, and at the external face a second Knowles microphone was fixed, so that the cell's gas length equals the wall thickness $(\sim 1.5 \mathrm{~mm})$. The Al foil was fixed in the inner part of the cavity using a conducting (silver) glue so that the distortion of the electric current distribution in the wall is minimized. Furthermore, care has also been taken to locate this second (reference) PA cell in the 
PA-SIGNAL

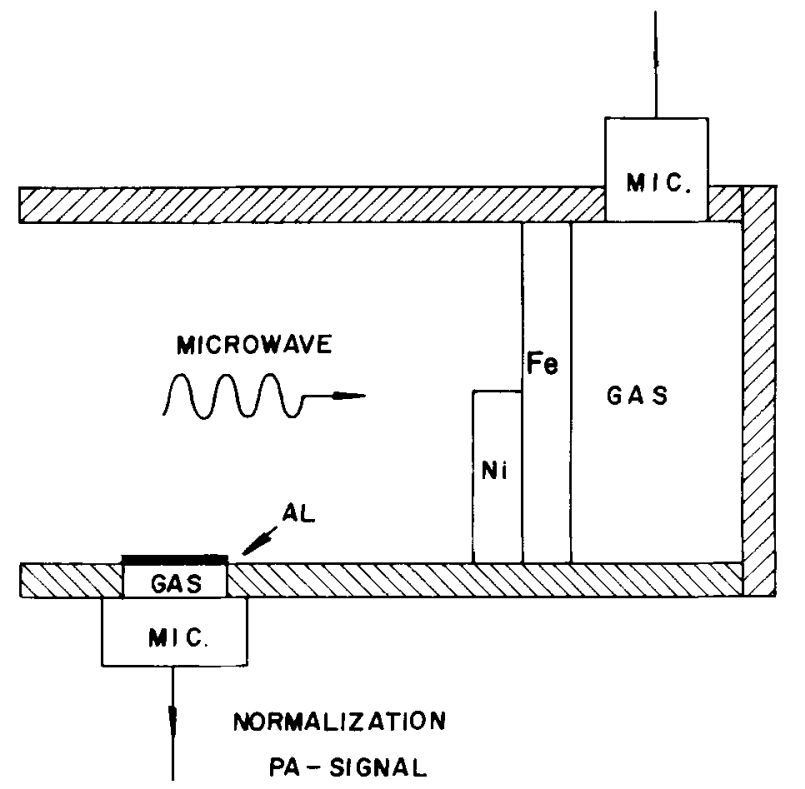

FIG. 1. Experimental PA setup showing the sample located at the end of a shorted waveguide. The sample is followed by a gas cell in which a miniature is mounted on one of its wall. A second cell was also built in one of the waveguide's wall so that the actual microwave magnetic field $H_{0}$ could be measured

region of maximum electric current distribution, namely, at $\lambda_{g} / 4=1.22 \mathrm{~cm}$ from the cavity end. In this way, we could measure the actual microwave magnetic field $H_{0}$ inside the cavity, which provided us the necessary normalization factor for the PA signal from our sample, as follows. The PA signal resulting from the microwave absorption in the magnetic sample is proportional to ${ }^{2,12,13} H_{0}^{2} V \mu$, where $\mu$ is the magnetic permeability of our sample, which is a function of an external magnetic field. As the Al foil is diamagnetic its signal at the reference cell is simply proportional to $H_{0}^{2}$. Thus, dividing the sample signal by the reference cell signal one is then probing the magnetic permeability of our sample. As the sample which forms one boundary of the PA cell is mechanically thin, care has been taken regarding the influence of the thermally induced bending of the foil. This thermoelastic effect severely modifies the thermoacoustic yield of a transmitted thermal wave if the sample thickness is of the order of magnitude of the thermal diffusion length. Using a relation derived by Junge et al., ${ }^{15}$ the thermoelastic correction has been estimated. These contributions turned out to be less than one percent at low modulation frequencies and raised up to a few percent at about $200 \mathrm{~Hz}$.

To actually measure the phase shift between the $\mathrm{Ni}$ and $\mathrm{Fe}$ signals, the microphone output was fed into two conventional lock-in amplifiers, connected in parallel. The signals of these two lock-in amplifiers were first set in phase at $0 \mathrm{G}$ by adjusting them to the maximum signal. We next shifted, still at $0 \mathrm{G}$, one the lock-in signals to a $90^{\circ}$ out-of-phase with respect to the other. We have then recorded the in-phase $\left(S_{0}\right)$ and quadrature $\left(S_{90}\right)$ signals as a function of the slowly sweeping dc magnetic field. In Fig. 2, we show the dependence of the normalized PA signals $S_{0}$ and $S_{90}$, due to the irradiation of a 9-GHz microwave field, as a function of the magnetic field for three different modulation frequencies. We note from Fig. 2 that the signal $S_{0}$ exhibits a shift of its maximum from $400 \mathrm{G}$ at $10 \mathrm{~Hz}$ to $300 \mathrm{G}$ at a frequency of $200 \mathrm{~Hz}$. Moreover, on further increasing the modulation frequency, the peak of $S_{0}$ was observed to remain at $300 \mathrm{G}$ and no phase shift was observed. In contrast, the signal $S_{90}$ at 10 and $50 \mathrm{~Hz}$ exhibited a broad maximum around $1.1 \mathrm{kG}$, and

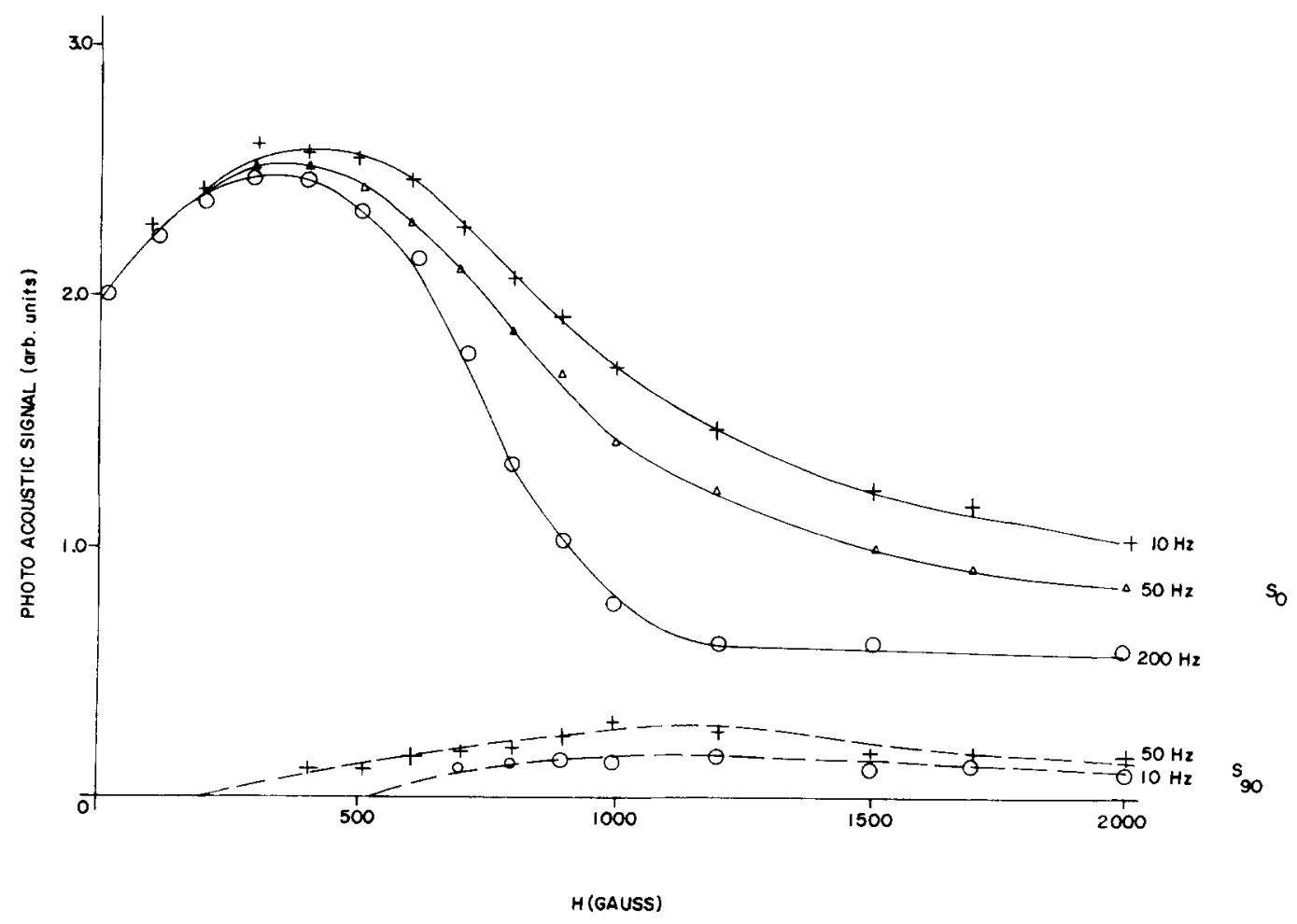

FIG. 1. Magnetic field dependence of the $0^{\circ}$ phase signal $S_{0}$ and the $90^{\circ}$ phase signal $S_{90}$ due to the absorption of $9-\mathrm{GHz} \mathrm{mi}-$ crowave, for several modulation frequencies. 


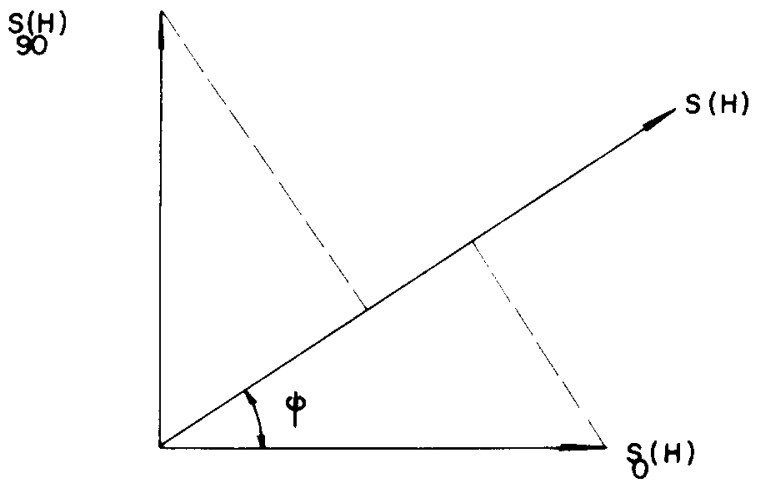

FIG. 3. PA signal at a phase $\psi$ expressed as the sum of the signals at $0^{\circ}$ and $90^{\circ}$ phases.

above $100 \mathrm{~Hz}$ the signal was vanishing small. These features, together with the fact that the $\mathrm{Fe}$ and $\mathrm{Ni}$ resonances are known ${ }^{13,16}$ to occur around 300 and $800 \mathrm{G}$, respectively, suggest that the signals $S_{0}$ and $S_{90}$ are somehow related to the separate contributions of $\mathrm{Fe}$ and $\mathrm{Ni}$, respectively. The positions of the resonances of the $\mathrm{Ni}$ and $\mathrm{Fe}$ foils were also checked by direct and independent FMR and PA measurements. That is, Fig. 2 suggests that $S_{0}$ is predominantly due to $\mathrm{Fe}$, whereas $S_{90}$ is predominantly due to Ni. To further pursue in the effort of sorting out the contributions of the two components we have then decided to look at the $50-\mathrm{Hz}$ signal and vary its phase as follows. For a given phase $\psi$ the PA signal produced in the gas cell can be written in terms of $S_{0}(H)$ and $S_{90}(H)$ as (cf., Fig. 3)

$$
S(H)=S_{0}(H) \cos \psi+S_{90}(H) \sin \psi .
$$

That is, the actual signal is the resultant of the $S_{0}$ and $S_{90}$, as depicted in Fig. 3. Thus, by varying the phase $\psi$, we have then searched for the phase at which only one component is present. This is shown in Fig. 4 in which the signal $S(H)$ given by Eq. (1) is plotted for $\psi=90^{\circ}, 60^{\circ}$, and $80^{\circ}$. We note from Fig. 4 that the signal for $\psi=-60^{\circ}$ exhibits only the iron resonance (at $300 \mathrm{G}$ ) whereas the signal for $\psi=80^{\circ}$ exhibits only the $\mathrm{Ni}$ resonance $(800 \mathrm{G})$. Furthermore, as the signal at $\psi=-60^{\circ}$ is only due to the iron, it is, of course, perpendicular to the nickel signal. We thus conclude that the nickel signal should be at angle of $30^{\circ}$ above $S_{0}$. Similarly, as the $\psi=80^{\circ}$ signal is only due to the nickel, we conclude that the iron signal is at $-10^{\circ}$ phase with respect to $S_{0}$. These results are depicted in the insert in Fig. 4 which shows that the actually measured signal $S_{0}$ is the resultant of the pure nickel and iron signals with an angle of $40^{\circ}$ between them. To check for this conclusion that the signal at $\psi=-60^{\circ}$ was only due to the iron contribution we have conducted a second test by correlating the signal $S_{0}$ at $200 \mathrm{~Hz}$ (which is certainly due to the iron component) with the signal $S$ at $50 \mathrm{~Hz}$, for several different phases. This is shown in Fig. 5, in which only for $\psi=-60^{\circ}$ is that the $50-\mathrm{Hz}$ signal equals the iron signal. This result proves, once again, that at a $-60^{\circ}$ phase we are unequivocally looking only at the iron contribution.

Finally, before concluding, we present a brief quantitative description of the above findings based upon the Rosencwaig and Gersho $(R G)^{17}$ one-dimensional thermal piston model. In Fig. 6 the geometry for the PA signal from the two foils is schematically depicted. As the penetration depth of the $9-\mathrm{GHz}$ microwave in the $\mathrm{Ni}$ and $\mathrm{Fe}$ foils is of the order of $1 \mu \mathrm{m}$, we describe the resulting heating sources at the rear

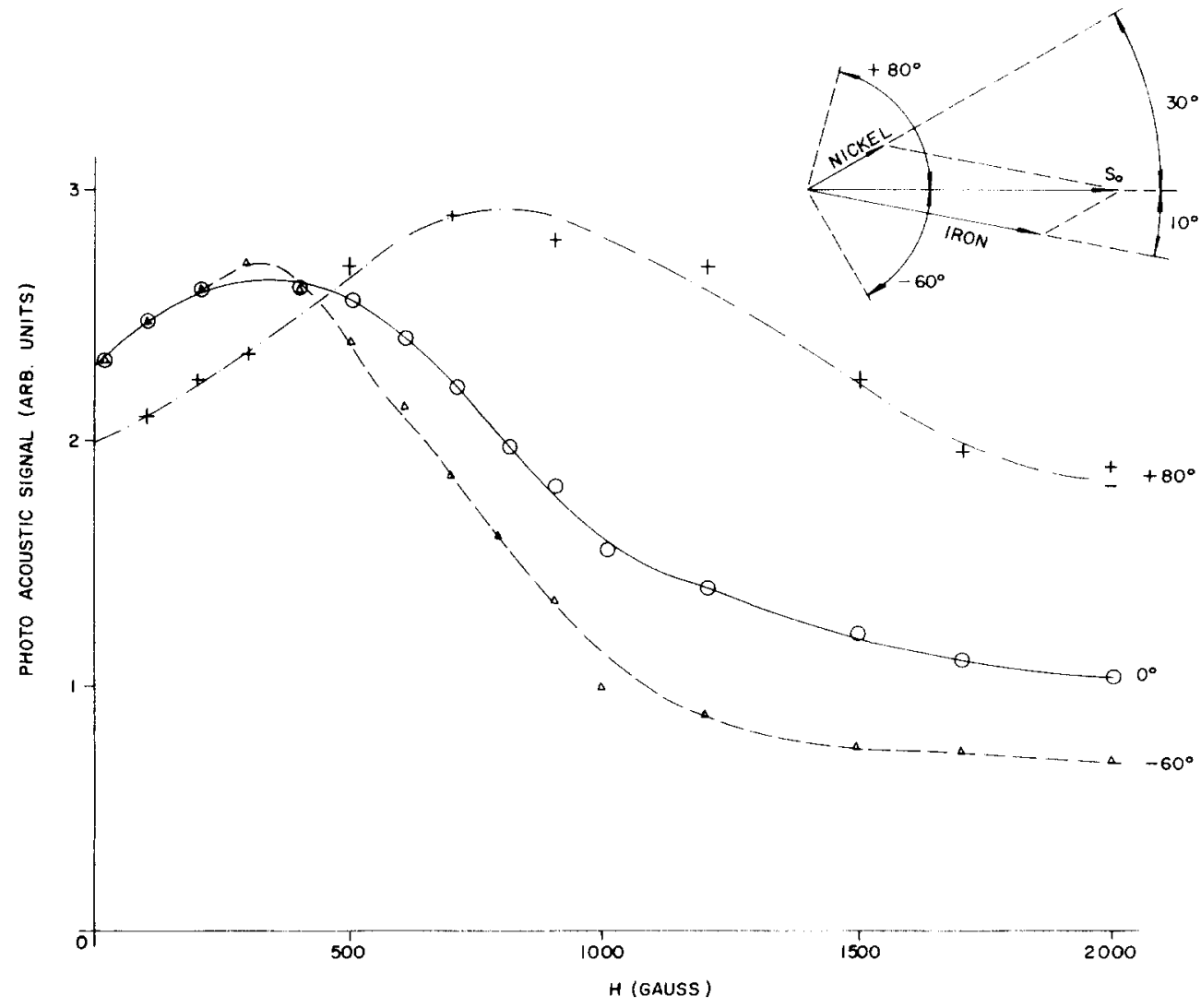

FIG. 4. Magnetic field dependence of the PA signal of our layered sample at three different phases $\psi=0^{\circ}$, $-60^{\circ}$, and $80^{\circ}$. The signal for $\psi=-60^{\circ}$ exhibits only the iron resonance at $300 \mathrm{G}$, whereas the signal at $80^{\circ}$ exhibits only the $\mathrm{Ni}$ resonance at $800 \mathrm{G}$. Modulation frequency 50 $\mathrm{Hz}$. 


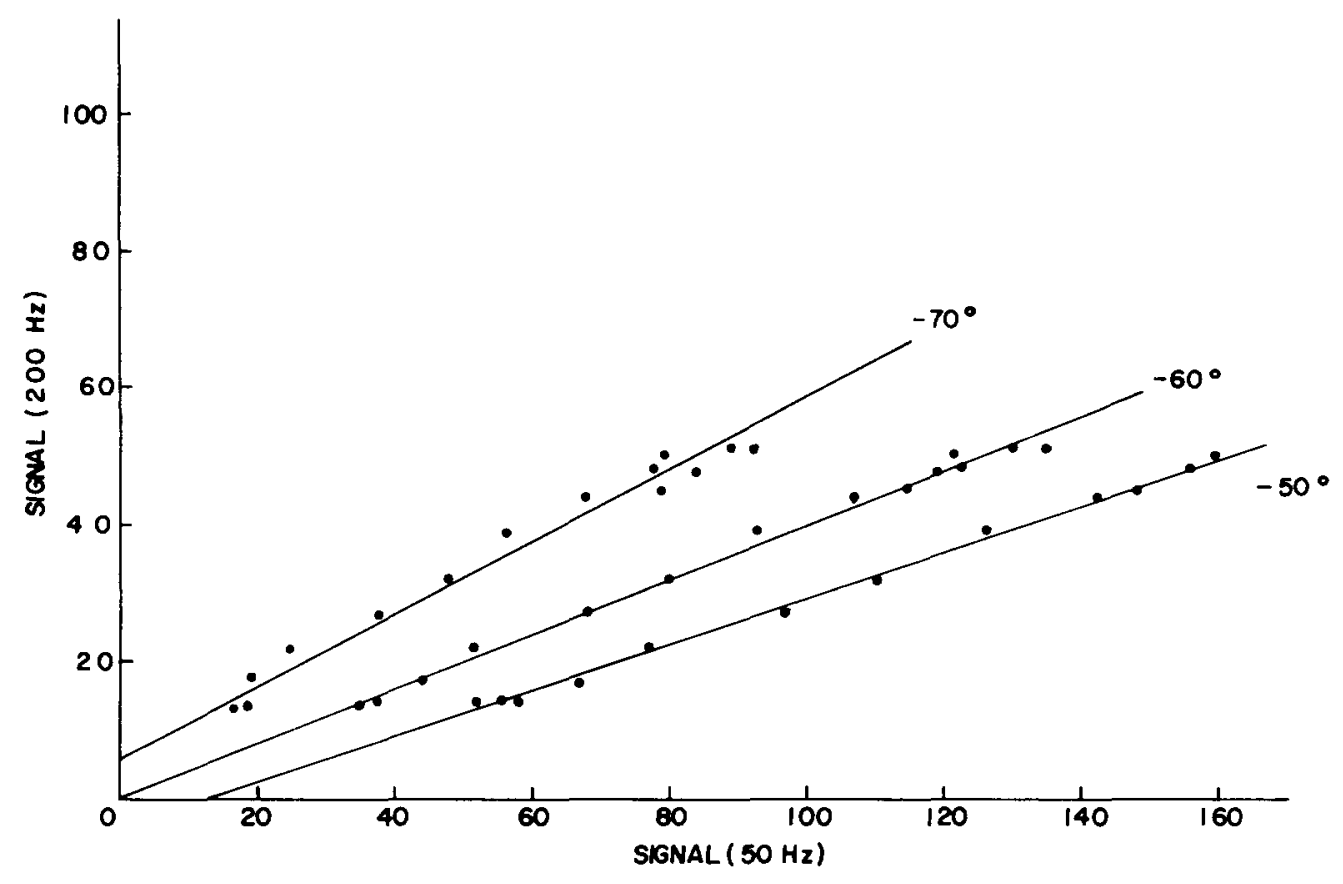

FIG. 5. Correlation of the signal $S_{0}$ at $200 \mathrm{~Hz}$ with the signal $S$ at $50 \mathrm{~Hz}$ for three different phases $-50^{\circ}$, $-60^{\circ}$, and $-70^{\circ}$, showing that only at $-60^{\circ}$ one has full correlation.

Ni-gas $\left(x=-l_{s}-l_{1}-l_{2}\right)$ and Fe-gas $\left(x=-l_{2}\right)$ interfaces by a surface absorption coefficient $\beta^{\prime}$. Thus, the periodical thermal power density generated at each foil [cf., Figs. 6(a) and $6(b)]$ may be written as

$$
\begin{aligned}
& \dot{H}_{\mathrm{Fe}}(x, t)=\beta_{\mathrm{Fe}}^{\prime} I_{0} \delta\left(x+l_{2}\right) e^{j \omega t}, \\
& \dot{H}_{\mathrm{Ni}}(x, t)=\beta_{\mathrm{Ni}}^{\prime} I_{0} \delta\left(x+l_{s}+l_{1}+l_{2}\right) e^{j \omega t},
\end{aligned}
$$

where $I_{0}$ is the incident microwave intensity, $l_{s}\left(l_{2}\right)$ the thick- ness of the $\mathrm{Ni}(\mathrm{Fe})$ foil, and $l_{1}$ the thickness of the glue layer bonding the two foils. Performing the usual calculations of the RG thermal piston model for the configurations of Fig. 6 , the temperature fluctuations $\theta$ at the sample-gas interface at $x=0$ responsible for the PA signal are found to be

$$
\theta_{\mathrm{Fe}}=\frac{2 r_{\mathrm{Fe}}^{\prime} I_{0}}{(1+g)^{2} e^{l_{1} \sigma_{2}}-(1-g)^{2} e^{-l_{2} \sigma_{2}}}, \quad r_{\mathrm{Fe}}^{\prime}=\frac{\beta_{\mathrm{Fe}}^{\prime}}{(k \sigma)_{\mathrm{Fe}}},
$$
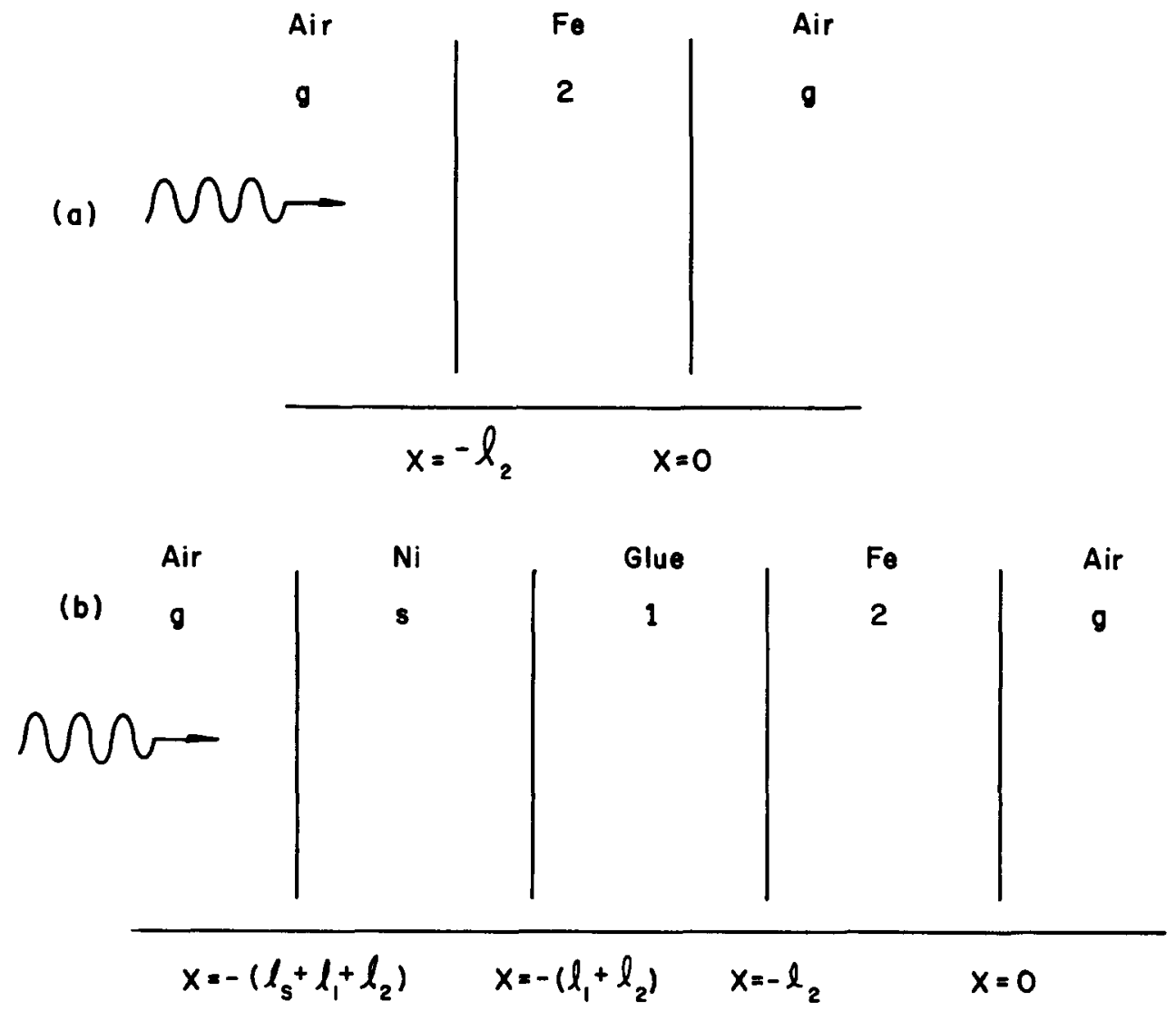

FIG. 6. Schematic geometry for the one-dimensional thermal piston model for the iron (a) and the nickel (b) PA signal generation. 
for the iron signal as in Fig. 6(a), and

$$
\theta_{\mathrm{Ni}}=-\frac{8 r_{\mathrm{Ni}}^{\prime} I_{0}}{A_{1} A_{3}+A_{2} A_{4}}, \quad r_{\mathrm{N} i}^{\prime}=\frac{\beta_{\mathrm{Ni}}^{\prime}}{(k \sigma)_{\mathrm{Ni}}},
$$

where

$$
\begin{aligned}
A_{1}= & (1-g)\left(1+b_{1}\right) \exp \left(-l_{s} \sigma_{s}-l_{1} \sigma_{1}\right) \\
& -(1+g)\left(1-b_{1}\right) \exp \left(l_{s} \sigma_{s}-l_{1} \sigma_{1}\right), \\
A_{2}= & (1-g)\left(1-b_{1}\right) \exp \left(-l_{s} \sigma_{s}+l_{1} \sigma_{1}\right) \\
& -(1+g)\left(1+b_{1}\right) \exp \left(l_{s} l_{s}+l_{1} \sigma_{1}\right), \\
A_{3}= & \left(1+\frac{b_{2}}{b_{1}}\right)\left(1-\frac{g}{b_{2}}\right) \exp \left(-l_{2} \sigma_{2}\right) \\
& +\left(1-\frac{b_{2}}{b_{1}}\right)\left(1+\frac{g}{b_{2}}\right) \exp \left(l_{2} \sigma_{2}\right), \\
A_{4}= & \left(1-\frac{b_{2}}{b_{1}}\right)\left(1-\frac{g}{b_{2}}\right) \exp \left(-l_{2} \sigma_{2}\right) \\
& +\left(1+\frac{b_{2}}{b_{1}}\right)\left(1+\frac{g}{b_{2}}\right) \exp \left(l_{2} \sigma_{2}\right) .
\end{aligned}
$$

In Eqs. (3)-(5) we have defined the following quantities: $\sigma_{i}=(1+j) a_{i}, \quad a_{i}=\left(\pi f / \alpha_{i}\right)^{1 / 2}, \quad g=k_{g} \sigma_{g} / k_{s} \sigma_{s}$, $b_{1}=k_{1} \sigma_{1} / k_{\mathrm{Ni}} \sigma_{\mathrm{Ni}}$, and $b_{2}=k_{\mathrm{Fe}} \sigma_{\mathrm{Fe}} / K_{\mathrm{Ni}} \sigma_{\mathrm{Ni}}$. The values of the thermal conductivity $k$ and the thermal diffusivity $\alpha$ for the different materials are $\mathrm{Ni}\left(k=0.91 \mathrm{~W} / \mathrm{cm}{ }^{\circ} \mathrm{C} ; \alpha=0.23\right.$ $\mathrm{cm}^{2} / \mathrm{s}$. $), \quad \mathrm{Fe}\left(k=0.81 \quad \mathrm{~W} / \mathrm{cm}^{\circ} \mathrm{C} ; \quad \alpha=0.23 \quad \mathrm{~cm}^{2} / \mathrm{s}\right)$, $\operatorname{air}\left(k=0.26 \times 10^{-3} \mathrm{~W} / \mathrm{cm}^{\circ} \mathrm{C} ; \alpha=0.22 \mathrm{~cm}^{2} / \mathrm{s}\right)$. Using these values for the physical parameters, it can be seen that, at 50 $\mathrm{Hz}$, both the $\mathrm{Ni}$ and the $\mathrm{Fe}$ foils are thermally thin so that Eqs. (3) and (4) may be approximated by

$$
\begin{aligned}
\theta_{\mathrm{Fe}} & \simeq \frac{\beta_{\mathrm{Fe}}^{\prime} I_{0}}{(k \sigma)_{\mathrm{Fe}}(l \sigma)_{\mathrm{Fe}}}, \\
\theta_{\mathrm{Ni}} & \simeq \frac{\beta_{\mathrm{Ni}}^{\prime} I_{0}}{(k \sigma)_{\mathrm{Ni}}(l \sigma)_{\mathrm{Ni}} \cosh \left(l_{1} \sigma_{1}\right)} .
\end{aligned}
$$

As the acoustic signal in the transducer gas is the sum of the contributions coming from the heat generated by the iron and the nickel absorption (i.e., $\theta=\theta_{\mathrm{Fe}}+\theta_{\mathrm{Ni}}$ ), it follows from Eqs. (6) and (7) that the ratio $R$ of the $\mathrm{Ni}$ to the $\mathrm{Fe}$ signals is

$$
R=\frac{\theta_{\mathrm{Ni}}}{\theta_{\mathrm{Fe}}}=\frac{\beta_{\mathrm{Ni}}^{\prime}}{\beta_{\mathrm{Fe}}^{\prime}} \frac{(k \sigma)_{\mathrm{Fe}}(l \sigma)_{\mathrm{Fe}}}{(k \sigma)_{\mathrm{Ni}}(l \sigma)_{\mathrm{Ni}}} \frac{1}{\cosh \left(l_{1} \sigma_{1}\right)}=A e^{-i \psi^{\prime}}
$$

where the phase angle $\psi$ between the two contributions is given by

$$
\tan \psi=\tanh \left(z_{1}\right) \tan \left(z_{1}\right)
$$

with $z_{1}=\left(\pi f l_{1}^{2} / \alpha_{1}\right)^{1 / 2}$. Equation (8) tells us that the phase difference between the $\mathrm{Ni}$ and the Fe signals comes from the time the heat wave, generated by the $\mathrm{Ni}$ absorption, takes to diffuse through the glue layer $(\sim 100 \mu \mathrm{m}$ thick). It also follows from Eq. (9) that, once we know the phase angle $\psi$, one can readily determine the glue thermal diffusivity, which, in most cases, is an unknown parameter. In our case $\left(\psi=40^{\circ}\right)$, the solution to Eq. (9) is $z_{1}=0.88$, which, for $f=50 \mathrm{~Hz}$ and $l_{1}=100 \mu \mathrm{m}$, gives $\alpha_{1} \simeq 0.02 \mathrm{~cm}^{2} / \mathrm{s}$. This value for the glue thermal diffusivity is quite comparable with the known values ${ }^{18}$ of the thermal diffusivity of cellulose acetate butyrate and vinyl butyrate, which are the commonly used active adhesive materials.

In conclusion, we have demonstrated in this paper that it is possible to use the phase shift of the acoustic response of the different constituents of a sample to the absorption of modulated radiation as a new method for photoacoustic microscopy. Even though the proposed method was demonstrated using ferromagnetic resonance, its generalization to other regions of the spectra is straightforward and is currently in progress in our laboratory.

The support by the Internationale Buero KFA, Julich, and by the CNPq is gratefully acknowledged.

'G. Diebold and D. L. McFadden, Appl. Phys. Lett. 29, 447 (1976).

${ }^{2}$ C. Evora, R. Landers, and H. Vargas, Appl. Phys. Lett. 36, 864 (1980).

${ }^{3}$ R. Melcher, Appl. Phys. Lett. 37. 895 (1980).

${ }^{4}$ A. Varson and A. M. Varson, J. Phys. D 14, L39 (1981).

'U. Netzelmann, E. V. Goldammer, J. Pelzl, and H. Vargas, Appl. Opt. 21, 1 (1982).

H. Coufal, Solid State Commun. 39, 467 (1981).

${ }^{7}$ R. C. DuVarney, A. K. Garrison, and G. Busse. Appl. Phys. Lett. 38, 675 (1981).

${ }^{8}$ W. Wettling, W. Jantz, and L. Engelhardt, Appl. Phys. A26, 19 (1981).

${ }^{4} \mathrm{H}$. Vargas, in Photoacoustics: Principles and Applications, edited by $\mathrm{H}$. Coufal (Vieweg, Berlin, 1981).

${ }^{1}$ U. Netzelmann, E. V. Goldammer, J. Pelzl, and H. Lechner, in Photoacoustics: Principles and Applications, edited by H. Coufal (Vieweg, Berlin. 1981).

'M. J. Adams and G. F. Kirkbright, Anal. 102, 281 (1977).

${ }^{2}$ C. L. Cesar, H. Vargas, J. Mendes, and L. C. M. Miranda, Appl. Phys. Lett. 43, 555 (1983).

${ }^{3}$ R. J. Von Gutfeld and R. L. Melcher, Appl. Phys. Lett. 30, 257 (1977); W, H. Wong, R. L. Thomas, and G. F. Hawking, Appl. Phys. Lett. 32, 538 (1978).

${ }^{4}$ M. L. Mackenthun, R. D. Tom, and T. A. Moore, Nature 279. $215\{1979\}$.

${ }^{15}$ K. Junge, B. Bein, and J. Pelzl, 3rd Topical Meeting on Photoacoustic and Photothermal Spectroscopy, Paris, 1983; J. Phys. Paris (to be published).

${ }^{10} \mathrm{~J}$. D. Jackson, in Classical Electrodynamics (Wiley, New York, 1975).

${ }^{17}$ A. Rosencwaig and A. Gersho, J. Appl. Phys. 47, 64 (1976).

${ }^{17} \mathrm{D}$. Grzegorczyk and G. Feineman, in Handbook of Plastics in Electronics (Reston, Reston, Virginia, 1974), p. 16-31. 